\title{
Protecting Data Privacy in Cloud
}

\author{
Masarath Begum, Mohammed Abdul Waheed
}

\begin{abstract}
Cloud is now widely used for the remote storage of data; it's an On-demand device and computer resource configuration process. This allows users to avoid locally saving and storing data. Remote data sharing is an inexpensive and effective way to share cloud users community resources. DiffieHellman used the previous approach to protect multi-owner cloud sharing for distributed groups. In the existing system, there is a community signature shared among all group members that contributes to the middle attack. The program suggested using the LFSR-dependent correlation method, which primarily used handshake protocol to safely exchange community signature to detect the attack, to detect an attack. If the calculated value exceeds one (value $>1$ ), the community's public key is changed to avoid abuse.
\end{abstract}

Keywords-Diffie-Hellman key Exchange, LFSR, Correlation

\section{INTRODUCTION}

Like Amazon, cloud can deliver powerful data centers to cloud users. Thus, users can use high-quality services as well as lower major infrastructural investments. Cloud computing is one of the most powerful tools for truly lowcost internet storage. Cloud computing is a computer based on the internet where all the common data, knowledge and applications are available on request to computers and devices. Many inventions allow the age of cloud computing, the automatic creation and use of computer technology. The storage of data is one of the most important services for cloud computing. In a company, its employees can store and share the cloud files in the same department or group [1]. The cloud helps staff to be freed from complicated storage and local data processing. On the other side, the security of the data stored in the cloud is highly risky for cloud storage. The confidentiality of the data held also poses a significant risk nonetheless [5]. This makes sure that the data stored in the cloud is secure. The massive implementation of cloud computing allows identity protection the biggest obstacle of cloud computing [7]. If the data protection of the identification is not ensured, customers may not want to link to cloud networks, because suppliers of cloud services and criminals may expose their individual identities quickly. Information disclosure plays a vital role in building confidence among customers and the service provider. Cloud customers love to utilize the cost efficiency functionality, but never want to disclose their private details.

Revised Manuscript Received on February 05, 2020.

* Correspondence Author

Masarath Begum1, Assistant Professor GNDEC, Bidar, Visvesvaraya Tecnological University Belagavi,Karnataka India

masrath456@gmail.com1

Dr.Mohammed Abdul Waheed, Associate Professor VTU CPGS, Kalaburgi, Visvesvaraya Tecnological University Belagavi, Karnataka India dr.mawaheed@gmail.com2

smoussa@cud.ac.ae

(C) The Authors. Published by Blue Eyes Intelligence Engineering and Sciences Publication (BEIESP). This is an open access article under the CC BY-NC-ND license (http://creativecommons.org/licenses/by-nc-nd/4.0/)

It requires delivering privacy to their personal data, each user acting as owner and dynamic data sharing [4]. This all is done ignoring the effects caused by cancelled cloud users. In the present work, each participant of a group can totally relish the applications provided by the cloud such as sharing and data storing.

\section{RELATED WORKS}

[1] E. Kallahalla clarified that Plutus is a cryptographic program for sharing files without trust on computer servers. This frame is used for cryptographic primitive materials to encrypt and share files. Plutus utilizes some methods to recover any user's ownership over their files. Plutus protocols aim at reducing the number of user-sharing encryption keys across file classes, splitting read and write file access, addressing user revocation and enabling an unconfused server to reach a file efficiently. The Open AFS project of Plutus. The test measurements show that Plutus promises a high overhead protection relative to devices that encrypt all network traffic. [2] Fiat and Naor have implemented a network multi-cast coordination program that involves multiple security risks. Consequently, secure multi-casts are built to prevent snooping and invasion. A professional system of main division is proposed here for the safeguarded coordination of complex parties. Using the IP multi-cast approach to reduce the opposing contact impact for the shortest possible time. To order to reduce traffic from rekeys, we often provide the proxy technique to reach the network manager of group members. [3] E. Modadugu N., Macham D. N. SiRius, a secure network file system are being launched by Boneh. SiRiUS also found that network storage was untrustworthy and had its own file level encrypted read-write access control. Key management and removal is simple without connection interactions. SiRiUS preserves the originality of the file system using the hash tree constructs. SiRiUS includes a new way to randomly navigate data without the use of a cryptographic file system block server. SiRiUS extensions offer large group sharing based on the NNL revoking link. SiRiUS is fantastic for the reliability of the underlying file system in cryptographic transactions. [4] G. Ateniese, K. Fu, M. Green, and S. Hohenberger, Strengthens proxy encryptions for securing dispersed storage. Here, the data owner uses exclusive and symmetric content keys for data. Later using the master public key it is additionally encrypted. The server uses proxy cryptography for access control to straightaway encrypt again the suitable content key(s) from the public key to an authorized user's key. Regrettably, linking any revoked user and untrusted server a collusion attack may occur, which may help to know the encrypted blocks key to decrypt it. [5] X. Liang and X. 


\section{Protecting Data Privacy in Cloud}

Lu make it crucial for the storage of data in cloud to document the possession of computing products and their operational background. In this paper it is proposed to tackle the neglected field of cloud computing with bilinear combination techniques by using a modern protected roots system. The scheme is crucial in providing information on confidential documents stored in the Cloud, in any unknown user automation on various data accesses and in monitoring the origin of controversial papers as well as the cloud computing after inquiries. In the standard model of proven security strategies, how the proposed system is covered is explained. [6] B. Waters proposes the new method to implement CIP-ABE (Standard Model Cyphertext-Political Attributes Cryption). It allows encryption or determines a computer property access control as a whole for an algorithm of authentication. The ciphertext time scales ' size, encryption and decryption correspond to the complexity of the formulation of the access to this system. A criticism of the common category layout was the only previous task of fulfilling these conditions. Group employees (customers) who store and provide other people with their own specific information in the cloud are a step towards reaching customers.

[7] Xuefeng Liu, Yuqing Zhang Boyang Wang, and Jingbo Yan proposes access acquiescence and data confident ability which is implemented using attribute-based encryption (ABE), lazy re-encryption, and proxy re encryption, the data or files are not shared in protected way among the users in the cloud. But in most of the existing system the revocation concept is not considered and when considered then overhead is too much. Because of not considering revoked member, we cannot identify whether the data accessed is by an authorized person or not.

\section{PROPOSED WORK}

In the previous existing system, we use Diffie-Hellman key interchange protocol to safely share the key between source and goal. However, as no party involved in the exchange is authenticated by its nature, the Diffie Hellman Key is susceptible to attacks in the middle [7]. We propose a solution called the LFSR-based Correlation algorithm with Hand-Shake for safe sharing of the system of group signatures. The following LFSR series shown in fig1.

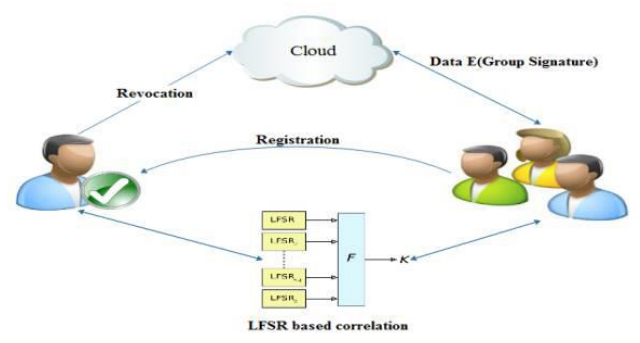

Fig 1: Architecture of proposed system

\section{SYSTEM DESIGN}

A comprehensive study of the use of Linear Feedback Shift Register (LFSR) by developers, programmers and researchers working in software, testing architecture and built-in self test environments is undertaken. For some of

the following reasons, LFSR are quite desirable systems for use in such environments, the configuration of LFSRs is straightforward and relatively standard. The change-over properties in the scanning design setting are easy to integrate. We are capable of creating a full and/or random vector and render them Primary candidates for their error detection and correctional property signature analysis framework. The geffe generator describes three LFSR maximum lengths whose lengths are relatively primitive L1, L2, L3 and nonlinear combinations $(\mathrm{f}(\mathrm{x} 1, \mathrm{x} 2, \mathrm{x} 3)=\mathrm{x} 1 \mathrm{x} 2=$ $\mathrm{x} 2 \mathrm{x} 3==\mathrm{x} 3 \mathrm{X} 1$ ) respectively.

1. Calculate the output series linear complexity.

2. Experimentally check the result.

3. Calculate the output correlation probability, $\mathrm{x} 1(\mathrm{t})$, and $\mathrm{x} 3(\mathrm{t})$.

4. Implement a function which recovers the initial condition of the first LFSR in view of a sufficient segment of the output sequence.

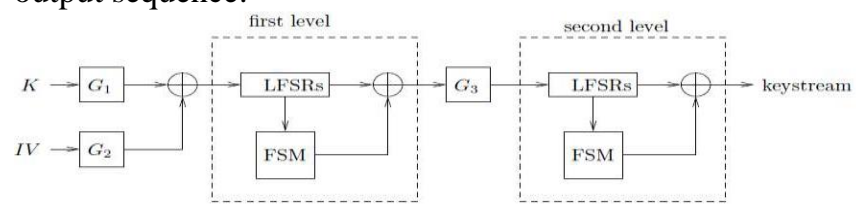

Fig 2: Working of Geffe generator with LFSR.

Where G1 and G2 are two transformations that are 128-bit tailored. The brain is empty on both terms. The generator is then 200 folded and a further stable G3 transformation occurs in the last 128 bits produced by the generator. The performance is then used for the second-level generator as a starting point, that is, the generator that generates the main stream as shown in fig2.

\section{DESIGN GOALS}

The following methodologies will be used for the design and implementation of algorithms.

- The Cloudsim 3.0.3 version can be used for performing simulation.

- Modify/Enhancing LFSR (Linear Feedback Shift Register) can be used for storing data on flip and flop basis.

Analysis of algorithms will be done after the simulation results. The simulation settings and parameters are changed according to the analysis of Register (LFSR) by developers, programmers and researchers working in software, testing architecture and built-in self test environments is undertaken. For some of the following reasons, LFSR are quite desirable systems for use in such environments, the configuration of LFSRs is straightforward and relatively standard. The change-over properties in the scanning design setting are easy to integrate. We are capable of creating a full and/or random vector and render them Primary candidates for their error detection and correctional property signature analysis framework.

The geffe generator describes three LFSR maximum lengths whose lengths are relatively primitive L1, L2, L3 and nonlinear combinations $(\mathrm{f}(\mathrm{x} 1, \mathrm{x} 2, \mathrm{x} 3)=\mathrm{x} 1 \mathrm{x} 2=$ respectively $\mathrm{x} 2 \mathrm{x} 3==\mathrm{x} 3 \mathrm{X} 1) .(2 \mathrm{~L} 2-001) .(2 \mathrm{~L} 3-1) .(1)$ 
1. Calculate the output series linear complexity.

- 2. Experimentally check algorithm.

The CloudSim framework provides platform assistance and increases the cloud condition, including a built-in memory, storeroom, containment communication and VM guide. Changed framework can be executed by a cloud office supporter [6]. In VM supply, consider the amplitude of different strategies. The user code layer revealed large segments, such as the machines calculation, their information, VMs, numerical clients, kinds and approaches. Furthermore, VMs host, working association and dynamic frame State screen are supported.

\section{METHODOLOGY}

The joint attack was introduced by the LFSR with some lengths. The assault on distinction is a splitting and winning strategy. This tries to restore the initial status of each LFSR regardless of the information of some main stream bits. In fact, the main bits can be identified by extracting the initialization using a thorough search made to initialize the right one, while the association between the respective sequence and the key stream is observed by computing.

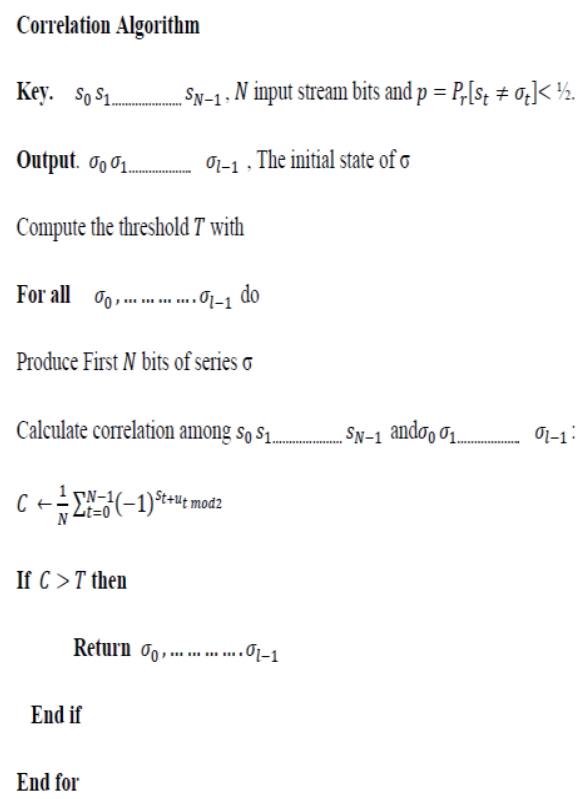

The initial state should evaluate all potential values if the two sequences are related. The correct value is determined by a classic hypothesis review, which refers to the correlation algorithm. The sequence can be determined in the same way as the key stream and the main bits are identified [3]. The following description focuses on binary sequences. In more depth, the internal condition of the generator at time $t$ can be divided into two size sections and modified separately for two features as in fig3.

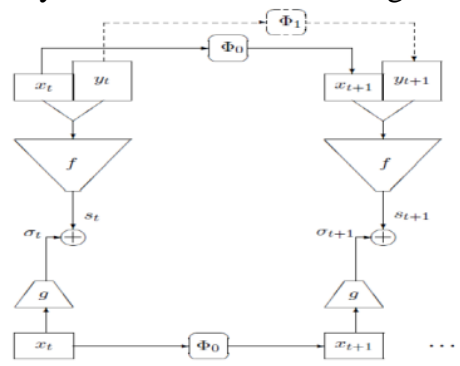

Fig 3: Model for the correlation attack
We created a correlation value in our proposed system to detect the mid-man-in attack. The meaning must be $0-1$ and that during contact it is not vulnerable to attack, and then it can be halfway targeted with a connection value $>1$. To overcome this issue, we request group managers to send you a new public key. We produced association worth to find Man medium spasm in our planned scheme. The value must be $0-1$ because it is not spasmed by broadcasting or message.

\section{RESULTS AND DISCUSSIONS}

CloudSim is a cloud environment simulation toolkit and a modeling, environment and application to estimate arrangement that can provide useful insight to discover such a powerful, massively circulated and scalable environment. General information of simulation parameters are as shown in table1.

Table 1

\begin{tabular}{|l|l|}
\hline \multicolumn{1}{|c|}{ PARAMETERS } & \\
\hline 1. Number of datacenters & 01 \\
\hline 2. Number of hosts & 01 \\
\hline 3. Number of processing units & 04 \\
\hline 4. Processing capacity(MIPS) & $\mathbf{9 , 6 0 0}$ \\
\hline 5. Storage Capacity & $11 \mathrm{~TB}$ \\
\hline 6. Total amount of RAM & $\mathbf{4 0 G B}$ \\
\hline 7. Broker policy & Round Robin \\
\hline
\end{tabular}

The graphs show the overall resource utilization and power consumption on the data centre.

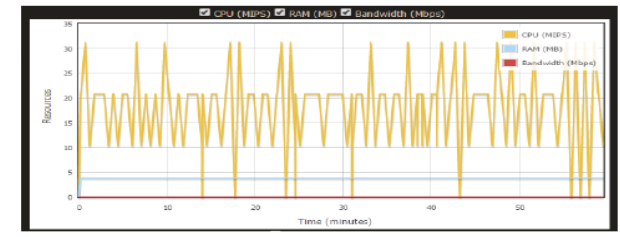

Fig 4: overall resource utilization in proposed approach

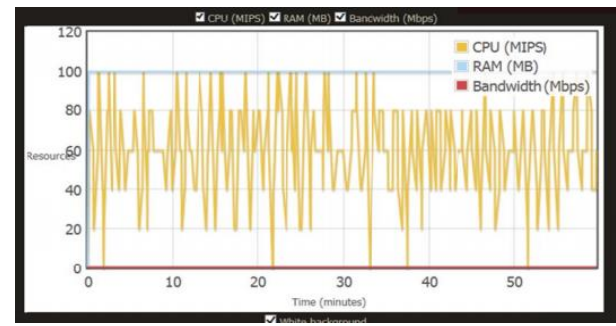

Fig 5: overall resource utilization in existing approach

\section{CONCLUSION}

There is no stability with the previous system MONA [7] as there are risks of the man-in - the-middle assault because the Diffie Hellman key exchange protocol is being used. We suggest a LFSR-based correlation attack to overcome this disadvantage. For addition, signatures will be produced that are popular throughout the community and can be randomly developed based on the time stamp signatures. Once we upload and save the script, we can have between them the caching layer. If the file is big we can partition and use the FTP algorithm to upload it part by part so that multiple threads can run simultaneously and the time required can be reduced. 


\section{ACKNOWLEDGMENTS}

Note that all success stories, satisfaction and euphoria that accompany successful performance of any task are incomplete without constant guidance or encouragement. I consider it a pleasure to express with all the people who led, supported and encouraged us in writing a book our appreciation and gratitude. I wish to express my sincere appreciation to Dr. Mohammed Abdul Waheed, my mentor, for providing us the means to effectively execute the document.

\section{REFERENCES}

1. M. Kallahalla, E. Riedel, R. Swaminathan, Q. Wang, and K. Fu, "Plutus: Scalable Secure File Sharing on Untrusted Storage," Proc. USENIX Conf. File and Storage Technologies, pp. 29-42, 2003.

2. A. Fiat and M. Naor, Broadcast Encryption, "Advances in Cryptology (CRYPTO)”, Proc. Intl Cryptology Conf. pp. 480-491, 1993.

3. E. Goh, H. Shacham, N. Modadugu, and D. Boneh, "Sirius: Securing Remote Untrusted Storage," Proc. Network and Distributed Systems Security Symp. (NDSS), pp. 131-145, 2003.

4. G. Ateniese, K. Fu, M. Green, and S. Hohenberger, "Improved Proxy Re-Encryption Schemes with Applications to Secure Distributed Storage", Proc.Network and Distributed Systems Security Symp. (NDSS), pp. 29-43, 2005.

5. R. Lu, X. Lin, X. Liang, and X. Shen, "Secure Provenance: The Essential of Bread and Butter of Data Forensics in Cloud Computing," Proc. ACM Symp. Information, Computer and Comm. Security, pp. 282-292, 2010.

6. B. Waters, "Ciphertext-Policy Attribute-Based Encryption: An Expressive, Efficient, and Provably Secure Realization,” Proc. Int'l Conf. Practice and Theory in Public Key Cryptography Conf. Public Key Cryptography, http://eprint.iacr.org/2008/290.pdf, 2008.

7. Xuefeng Liu, Yuqing Zhang Boyang Wang, and Jingbo Yan, "Mona: Secure Multi-Owner Data Sharing for Dynamic Groups in the Cloud", IEEE Tranction On Parallel And Distributed Systems, 2013.

\section{AUTHORS PROFILE}

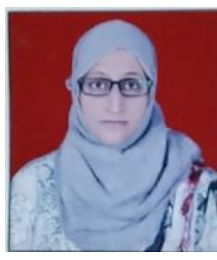

Masrath Begum, received B.E and M.Tech degree in CSE from Khaja Banda Nawaz Engineering College, Kalaguragi, Karnataka. Pursuing Ph.D from VTU Belagavi. Presently working as Assistant Professor CS\&E Department GNDEC, Bidar, Karnataka.

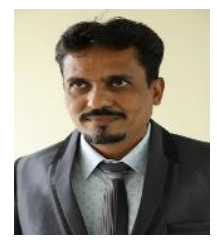

Dr. Mohammed Abdul Waheed, pursued B.E and M.E degree from Khaja Banda Nawaz College of Engineering and completed Ph.D in the year 2012 Presently working as Associate professor, Department of Computer Science \& Engineering, Visvesvaraya Technological University Centre for PG Studies, Kalaburagi, Karnataka, India. 20 years of academic experience, $4 \mathrm{Ph} . D s$ completed, 5 are pursuing. Area of interest for research Computer Networks, Cloud computing, MANET, WSN and published more than 100 papers in journals and conferences. 